
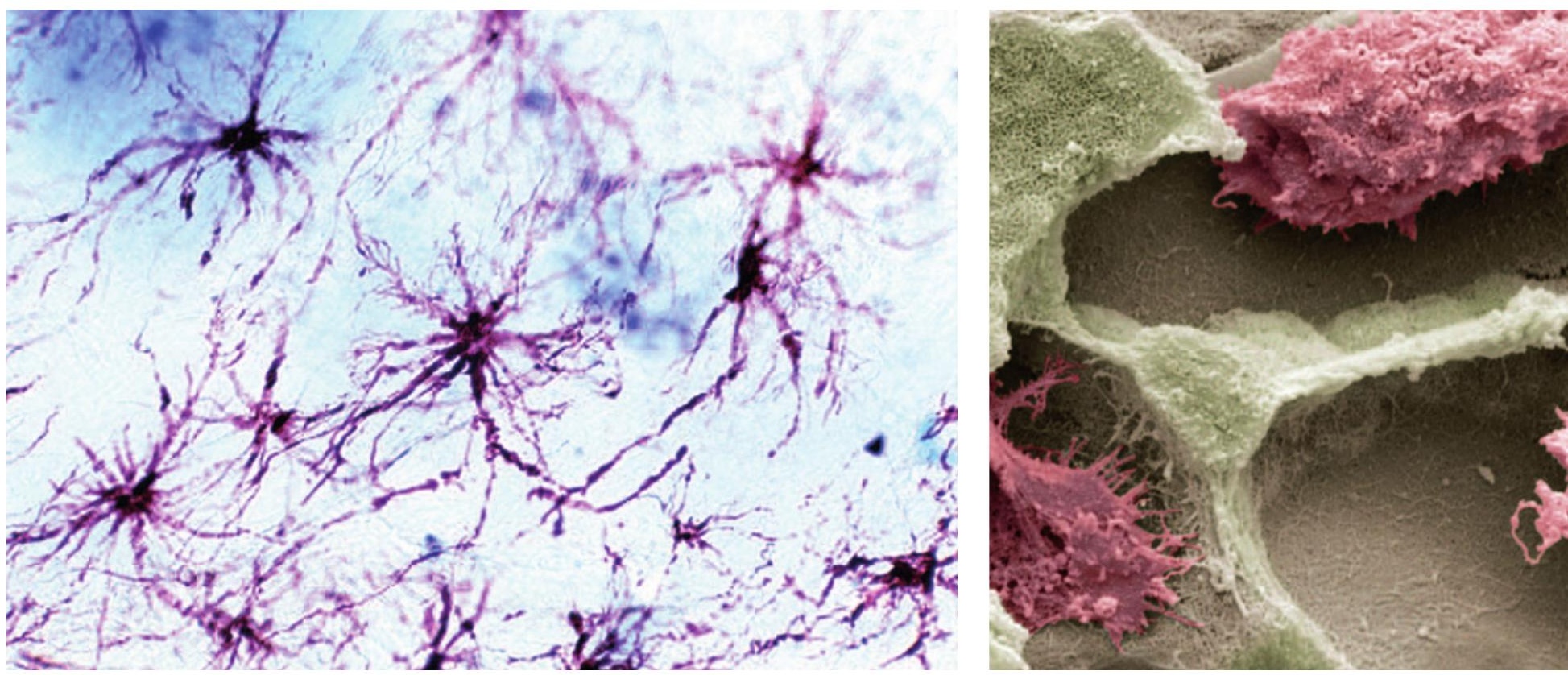

Osteoblasts (purple) are cells that form bones in healthy individuals (left). Myeloma promotes the activity of osteoclasts (right), which cause bone degradation.

\title{
The path to disease
}

\section{Multiple myeloma begins with a benign condition before progression to full-blown cancer, and work is underway to uncover the origins of both.}

\section{BY CYNTHIA GRABER}

$\mathrm{M}$ ultiple myeloma takes root years before any symptoms appear. Some plasma cells avoid the normal checks in the bone marrow and begin to clone themselves and proliferate, a condition known as 'monoclonal gammopathy of undetermined significance' (MGUS), which can persist for years or even decades. It might begin to weaken bones and cause some protein deposits on organs, but the patient typically never feels a thing. In multiple myeloma, however, these same monoclonal plasma cells explode in numbers and become malignant, causing anaemia, bone destruction and kidney failure.

Only about $1 \%$ of people with MGUS develop full-blown multiple myeloma each year. But several questions remain, with implications for early intervention and treatment. Most importantly, what causes MGUS to develop in the first place? And what is it that catapults the quiescent MGUS cells into the deadly disease of multiple myeloma?

\section{THE ORIGINAL CAUSE}

The origins of MGUS are mysterious, although researchers have been able to tease out a variety of risk factors. Men seem to be at higher risk than women. Individuals whose immune systems have been suppressed by autoimmune diseases seem to develop the disease at greater numbers, as do people over the age of 50. Some of these trends may be due to the body's heightened effort (in the case of diseases) or long-term struggle (in the case of age) to produce cells to fight infection, until finally one mutates and proliferates. "If you are older, the plasma cells are more likely to be responding to antigenic stimulation [such as an infection] all the time," explains haematologist Vincent Rajkumar, a multiple myeloma specialist at the Mayo Clinic in Rochester, Minnesota, "and at some point a mistake is going to happen."

Another likely cause is genetics. There is a greater risk of developing MGUS if an immediate family member has the disease, although it is not clear whether this is due to genetics, the environment or a combination of the two. And several studies have shown that African-Americans contract MGUS at roughly twice the rate as Caucasians, after adjusting for potential socioeconomic and environmental effects.

Environmental connections are also suspected in both MGUS and multiple myeloma. A few years after the attacks on 11 September 2001, researchers tasked with evaluating the health impacts of the fires and dust from the World Trade Center alerted the public that a number of first responders had contracted multiple myeloma. A statistically significant link to the event has not held up over time, however. Despite this lack of a convincing connection, epidemiological studies have focused on whether there is a link with specific chemicals, or classes of chemicals. Some studies suggest that firefighters generally seem to contract multiple myeloma at higher rates, and farm workers may too.

According to a Mayo Clinic review published in 2010, the incidence of MGUS rises with increased pesticide exposure. Rajkumar, one of the review's authors, highlights exposure to benzene, petrochemicals and pesticides as factors that have been shown to place people at higher risk, suggesting that these chemicals may lead to DNA mutations that could convert normal plasma cells into malignant ones.

But W. Michael Kuehl, who studies the causes of multiple myeloma at the National Cancer Institute (NCI) in Bethesda, Maryland, maintains that the environmental triggers have not yet been proven. "It's very hard to make sure that the control group and the 'at risk' group are exactly the same" in epidemiological studies, he says.

Brian Durie, a myeloma specialist at the Cedars-Sinai Outpatient Cancer Center in Los Angeles, California, thinks the environmental impact may be significant, particularly for certain classes of herbicides and pesticides. "There are
ONATURE.COM For some of the latest research on multiple myeloma: go.nature.com/9dda9p 
